\title{
Reporting Guidelines for Music-based Interventions
}

\author{
Sheri L. Robb, PhD, MT-BC, \\ School of Nursing, Indiana University
}

Debra S. Burns, PhD, MT-BC, and

Department of Music and Arts Technology, School of Engineering and Technology, Indiana University-Purdue University Indianapolis

Janet S. Carpenter, PhD, RN, FAAN

Department of Adult Health, School of Nursing, Indiana University

\begin{abstract}
Music-based interventions are used to address a variety of problems experienced by individuals across the developmental lifespan (infants to elderly adults). In order to improve the transparency and specificity of reporting music-based interventions, a set of specific reporting guidelines is recommended. Recommendations pertain to reporting seven different components of music-based interventions including intervention theory, intervention content, intervention delivery schedule, interventionist, treatment fidelity, setting, and unit of delivery. Recommendations are intended to support CONSORT and TREND statements for transparent reporting of interventions while taking into account the variety, complexity, and uniqueness of music-based interventions.
\end{abstract}

\section{Keywords}

music therapy; music; reporting guidelines; evidence-based practice

\section{INTRODUCTION}

\begin{abstract}
Music-based interventions in health care are generally considered low risk and palatable to patients of various ages from infants to elderly. While there is a strong intuitive appeal to most patients and healthcare providers regarding the use of music in healthcare environments, the evidence to support its integration into healthcare is equivocal. For example, a recent Cochrane review suggests that the influence of music on pain relief is highly variable, with tentative clinical importance (Cepeda, Carr, Lau, \& Alvarez, 2006). Studies exploring the use of music to decrease anxiety are also inconclusive with results depending on intervention type (Pelletier, 2004). Integrative reviews conclude that divergent results are the result of methodological problems across studies (Evans, 2002; Pothoulaki, 2006). In particular, many studies lack sufficient description of music interventions to enable cross-study comparisons, generalization, and integration into practice.

Detailed intervention research reporting is essential to interpretation, replication, and eventual translation of music-based interventions into practice. The Consolidated Standards for Reporting Trials (CONSORT) and Transparent Reporting of Evaluations with Nonrandomized Designs (TREND) statements, each with multiple item reporting checklists, were developed to help improve the quality of research reports (Altman, et al., 2001; Des
\end{abstract}

Contact Information: Sheri L. Robb; Indiana University School of Nursing; 1111 Middle Drive, NU 492A; Indianapolis, IN 46202-5107. Phone: 317-274-3152. Fax: 317-278-2021. shrobb@iupui.edu. 
Jarlais, Lyles, \& Crepaz, 2004; Schulz, Altman, \& Moher, 2010). One specific item on CONSORT and TREND checklists is dedicated to intervention reporting, but limited in detail. Subsequent publications have explored complexities related to transparent reporting of behavioral and non-pharmacological interventions, especially in the area of intervention reporting (Altman, et al., 2001; Boutron, et al., 2008a, 2008b; Des Jarlais, et al., 2004; Dijkers, et al., 2002; Marks, 2009, 2010; Mayo-Wilson, 2007; Perera, Heneghan, \& Yudkin, 2007; Schulz, et al., 2010).

Music-based interventions are especially difficult to fully and transparently describe because of the complexity of music stimuli and other factors such as choice of music, mode of delivery, or the combination of music with other intervention strategies. In a recent review, Robb and Carpenter (2009) identified 11 intervention components unique to music-based interventions. Despite the publication of elaborated CONSORT guidelines to describe intervention reporting complexities for non-pharmacologic treatment, herbal interventions, and behavioral medicine, there remain many issues specific to music-based intervention reporting that have not been addressed (Boutron, et al., 2008a, 2008b; Davidson, et al., 2003; Gagnier, et al., 2006). Given the variety and complexity of music-based interventions, we are recommending specific reporting guidelines to improve the transparency and specificity of reporting music-based intervention research. The proposed guidelines build on recommendations from CONSORT and TREND, with evidence-based arguments for the inclusion of specific information when reporting music-based interventions.

\section{FORMULATION OF REPORTING GUIDELINES}

The proposed recommendations focus on the area dedicated to interventions in the CONSORT and TREND guidelines (see Table 1). Our recommendations were formulated based on our systematic review of music-based intervention reporting (Robb \& Carpenter, 2009) and on broader-based reviews of behavioral intervention reporting (Boutron, et al., 2008a, 2008b; Dzewaltowski, et al., 2004; Gagnier, et al., 2006; Lechago, Carr, Lechago, \& Carr, 2008; Mayo-Wilson, 2007; Moher, Jones, \& Lepage, 2001). We started by examining the 11 intervention components identified by Robb and Carpenter (2009) and generated an extensive list of reporting criteria.

Next we condensed the 11 components to seven using the criteria that reported information must be: 1) relevant across a wide range of music-based interventions; 2) essential for interpretation of outcomes; and 3) necessary for replication and translation. The following section presents our recommendations for music-based intervention reporting, along with an evidence-based rationale for inclusion of each reporting variable. Table 2 provides a concise overview of the seven criteria and is intended to be used as a checklist by investigators to verify that each criterion is addressed in published reports of music-based interventions.

\section{RECOMMENDATIONS FOR MUSIC-BASED INTERVENTION REPORTING}

Item 4A: Intervention Theory. Provide a rationale for the music selected; specify how qualities and delivery of the music are expected to impact targeted outcomes.

Our previous reviews indicate that many investigators do not provide rationale for use of music as an intervention. Although some authors provided a theoretical conceptualization of the problem, they did not describe the mechanistic or mediating variables explaining how the music was expected to or actually influenced specified outcomes. An atheoretical approach can become problematic when interpreting research results or performing crossstudy comparisons. For example, music listening interventions are frequently used to decrease acute and/or chronic pain in cancer patients throughout the treatment trajectory (Cepeda, et al., 2006). Pain is a multi-dimensional phenomenon and different dimensions 
may be more or less amenable to change with music-based interventions. Though music listening interventions seem to be somewhat beneficial in reducing pain (Cepeda, et al., 2006), results are mixed, perhaps due at least in part to differences in measurement of pain outcomes. A clear theoretical conceptualization with a proposed mechanism of action for music can help guide selection of outcome measures, which would in turn clarify any beneficial effects of music. Thus, we recommend that investigators provide a rationale for the music selected and specify how qualities and delivery of the music are expected to impact targeted outcomes.

Item 4B: Intervention Content. Provide precise details of the music intervention and, when applicable, descriptions of procedures for tailoring interventions to individual participants.

Item 4B.1: Who Selects the Music. Specify who selected the music: (1) preselected by investigator, (2) participant selected from limited set, (3) participant selected from own collection, or (4) tailored based on patient assessment.

Several meta-analytic and comparative studies have explored the potential influence of who selects music on intervention effectiveness; disparate findings support arguments for reporting this aspect of music-based interventions (Allen \& Blascovich, 1994; Labbe, et al., 2007; Pelletier, 2004; Potteiger, Schroeder, \& Goff, 2000; Silverman, 2003). Increased understanding about who selects music and the influence on health outcomes will provide clinically relevant information about delivery methods and related cost. However, meaningful cross-study comparisons and meta-analytic studies are difficult in the absence of clear reporting. A Medline search using the terms "self-selected music," "patient-selected music," and "participant-selected music" published between 2000 and 2009 resulted in 15 publications. Inspection of each article revealed more detailed information about how the term "participant/patient/self-selected music" was actually defined: 1) six studies (40\%) used music from the participants own collection (i.e., they were asked to bring music from home or could request any piece of music) (Ebneshahidi, Mohseni, Ebneshahidi, \& Mohseni, 2008; Hernandez-Ruiz \& Hernandez-Ruiz, 2005; Labbe, et al., 2007; Sarkamo, et al., 2008; Simcock, et al., 2008; Wang, Kulkarni, Dolev, \& Kain, 2002); 2) six studies (40\%) asked participants to choose music they preferred from a limited set of investigatorselected music(Allen \& Blascovich, 1994; Buffum, et al., 2006; Chlan, Tracy, Nelson, \& Walker, 2001; Hayes, et al., 2003; Kang, et al., 2008; Smolen, et al., 2002); 3) two studies (13\%) did not provide a clear enough description to determine how participant selected music was defined (Lee, et al., 2004; Potteiger, et al., 2000); and 4) one study (7\%) tailored music based on patient assessment (Clark, et al., 2006). These data suggest the need for clearer terminology when reporting the use of participant-selected music and new terminology to capture the tailored selection of music.

Tailoring is defined by Kreuter and Skinner (2000) as "any combination of information or change strategies intended to reach one specific person, based on characteristics that are unique to that person, related to the outcome of interest, and have been derived from an individual assessment." Although tailored interventions appear frequently in music therapy clinical practice, they have not been widely tested through controlled research - but have begun to appear in published literature (Enderlin \& Richards, 2006; Okamoto, et al.; van der Geer, et al., 2009).

We recommend that investigators specify who selected the music for their study using the following 4 categories: 1) pre-selected by investigator, 2) participant selected from limited set (i.e., the participant is offered a choice of music from a list that was pre-selected by the investigative team), 3) participant selected from own collection (i.e., the participant brought music from home or was given the opportunity to request any piece of music), and 4) 
tailored based on participant assessment (i.e., music is selected or created for each participant based on specified criteria).

Item 4B.2: Music. When using published music, provide reference for sheet music or sound recording.

When using improvised or original music, describe the music's overall structure (i.e., form, elements, instruments, etc).

Investigators must consider numerous qualities of music when designing and testing interventions including form, tempo, rhythm, melody, harmony, voicing, and tonality. For example, we know that changes in loudness, especially increases or sudden decreases in dynamics, will elicit attentive responses in the listener that are also associated with physiological arousal (Huron, 1992; Thaut, 2002, 2005). In contrast, sustained or limited changes in dynamics can lead to auditory habituation and diminished arousal - where the music becomes background (Huron, 1992; Thaut, 2002, 2005). In this example, eliciting or diminishing attention and arousal in the listener depends on the compositional features of the music; therefore, it is not enough to cite the title or genre of the composition. For instance, Pachelbel's Canon in D major has been used in studies seeking to elicit a relaxation response (Allen \& Blascovich, 1994; Knight \& Rickard, 2001). However, there are hundreds of recordings and arrangements that vary significantly in tempo and instrumentation - these include orchestral, string quartet, solo guitar, vocal, and heavy metal arrangements. Such variations likely contribute to disparate responses in listeners and final outcomes. Detailed reporting allows cross-study comparisons and better replication of findings. As such, we recommend that authors provide titles of music, including a reference for sheet music or sound recordings. In cases where original or improvised music is used, authors are encouraged to describe musical elements (e.g., tempo, rhythmic structure, form), especially those that are expected to impact targeted outcomes.

Item 4B.3: Music Delivery Method (Live or Recorded). When using live music, specify who delivered the music and the size of the performance group (e.g., interventionist only, interventionist and participant).

When using recorded music, specify placement of playback equipment and the use of headphones vs. speakers. Specify who determined/controlled volume (e.g., interventionist; participant). Specify decibel level of music delivered and/or use of volume controls to limit decibels.

A majority of authors report whether music delivered to study participants was live or recorded, but leave out more detailed information that is relevant to study interpretation and replication. Music perception studies indicate that factors such as musical training, gender, and the complexity of a musical arrangement (i.e., polyphonic vs. homophonic music) can have an impact on neurological processing of a sound stimulus; therefore, when using live music we recommend that authors specify who is delivering the music and the size of the performance group.

When using recorded music, investigators often overlook the importance of reporting details about how the music was delivered (i.e., headphones vs. speakers) and the volume of the music. The use of speakers or headphones can create a different listening experience that may positively impact intervention effects. For example, several studies have used headphones to block external, environmental sounds that can heighten anxiety or distract participants from the intervention (Fowler-Kerry \& Lander, 1987; Hatem, Lira, \& Mattos, 2006; Megel, Houser, \& Gleaves, 1998; Noguchi, 2006; Robb, Nichols, Rutan, Bishop, \& Parker, 1995). Other studies have delivered music using speakers to allow the participant to attend to both the music and other aspects of the environment. For example, Grasso and colleagues (2000) used music to structure chest physiotherapy exercises for children with 
cystic fibrosis. The music was played from a speaker so that both the parent who delivered the exercises and the child could hear the music. Reporting these variables allows a more thorough examination of factors that may be responsible for intervention effects.

Second, the volume of the music, which is measured in decibels, is important to consider especially in terms of auditory health. Volume of auditory stimuli has been positively correlated with arousal levels experienced by the music listener (Huron, 1992). Reporting whether the investigator or the participant selects/controls the volume of music during the listening experience is an important factor to consider when evaluating and translating study outcomes. Volume limits on playback equipment allow the investigator to control for decibel level of presented stimuli and prevent unintended exposure to unhealthy sound levels. This is especially important when introducing additional sound stimuli in settings where noise levels may be particularly relevant for participant safety, as is the case with premature infants, individuals with dementia, or others (Dewing \& Dewing, 2009; Graven, 2000; Philbin \& Klaas, 2000; Ragneskog, Gerdner, Josefsson, \& Kihlgren, 1998; Sloane, et al., 2002).

When using recorded music, in addition to reporting the type of playback equipment used (see Item 4B.4), we recommend that investigators also report the following information when applicable: 1) whether music was delivered using speakers or headphones, 2) placement of speakers, 3) who determined/controlled the volume of the music, and 4) the decibel ( $\mathrm{db}$ ) level of the music delivered and/or use of volume controls to limit db levels.

Item 4B.4: Intervention Materials. Specify music and/or non-music materials.

The vast majority of music-based intervention studies use materials other than the musical selections that are played or performed for study participants (Robb \& Carpenter, 2009); therefore, any materials used for intervention delivery should be clearly identified and described. The term music material refers to musical instruments or items used to create musical sounds. Non-music materials refer to audio-playback equipment, headphones, or other non-musical items, such as illustrated books or tip sheets that were an integral part of the intervention. In the case of music instruments, the quality, timbre, and resonance of instruments vary greatly. For example, a six-string acoustic guitar with steel strings has a different timbre (sound quality) and is generally louder than a six-string classical guitar with nylon strings. Investigators cannot determine if these factors matter unless they get reported. Similarly, non-musical materials, such as audio playback equipment, visual or tactile aids, and/or printed material, can vary in quality, content, and composition. We recommend that authors list all materials by name and when possible include brand, model number, quantity, and descriptive information about all intervention materials. Reporting precise details allows for easier replication and evaluation about sensory stimuli introduced into the study environment.

Item 4B.5: Intervention Strategies. Describe music-based intervention strategies under investigation (examples: music listening, songwriting, improvisation, lyric analysis, rhythmic auditory stimulation, etc).

Although categorical descriptions of music-based interventions, such as music-assisted relaxation, songwriting, or music imagery, are frequently reported, the level of detail about categorical components varies greatly across studies. For example, categorical descriptions such as "music-based relaxation" or "music relaxation" strategies have been used to describe studies that combined music with progressive muscle relaxation, diaphragmatic breathing, imagery, or simply listening to music(Robb \& Carpenter, 2009). This illustrates that simple categorical labels do not provide enough specificity to adequately describe an intervention, especially when the intervention uses multiple strategies or components. Terms may be defined differently by investigators, leading to different interventions with the same label. 
Although such labels help investigators and clinicians categorize interventions, without additional information these categorical descriptions create a level of ambiguity that hinders or prohibits interpretation and replication of study findings. Incomplete information about the intervention often diminishes clinicians' ability to use study findings to inform practice and can lead to exclusion of studies in meta-analyses and literature reviews. We recommend that authors categorize and fully describe the content and procedural steps for each intervention component, such that the reader can fully interpret study findings.

Item 4C: Intervention Delivery Schedule. Report number of sessions, session duration, and session frequency including practice sessions.

Our review indicated consistent reporting about the number of sessions delivered; less consistent was reporting about session duration and frequency (i.e., how sessions were spaced over time). Information about duration and frequency of session administration is essential when evaluating studies, especially in terms of dosage. The number, duration, and frequency of sessions necessary to derive clinical benefit are questions of paramount importance to clinical practice. For example, a previous study with bone marrow transplant patients suggests that more music sessions are positively correlated to improvements in mood (Cassileth, Vickers, \& Magill, 2003). Meta-analytic studies often seek to answer questions about treatment dose, but these questions are often left unanswered due to incomplete reporting. We recommend that authors report information about intervention delivery in all three areas: number, duration, and frequency of sessions.

Item 4D: Interventionist. Specify interventionist qualifications and credentials. Specify how many interventionists delivered study conditions.

Music-based interventions are delivered by a variety of professionals, with varied levels of training in music and/or the therapeutic application of music. It is important that authors report qualifications of individuals delivering an intervention, including professional credentials, because this information has implications for eventual translation of research to practice and future studies that may investigate the level of expertise required to effectively deliver an intervention. One meta-analytic study already suggests that interventionist qualifications may be related to music-based intervention outcomes (Standley, 2000). In addition, the use of multiple trained interventionists should be reported as this strengthens arguments for an intervention effect, rather than a person effect.

Item 4E: Fidelity Strategies for Treatment Delivery. Describe strategies used to ensure that treatment and/or control conditions were delivered as intended (e.g., interventionist training, manualized protocols, and intervention monitoring).

Interventionist training and intervention monitoring are two important aspects of treatment fidelity (Moncher, 1991; Resnick, et al., 2005; Santacroce, Maccarelli, \& Grey, 2004). These procedures help to ensure that interventions are delivered consistently and accurately across study participants, yet treatment fidelity strategies are rarely reported in music-based intervention studies (Robb \& Carpenter, 2009). The National Institutes of Health Behavior Change Consortium has published recommendations to encourage more consistent incorporation of treatment fidelity strategies into behavioral intervention research across five areas: 1) study design, 2) provider training, 3) treatment delivery, 4) treatment receipt, and 5) enactment of treatment skills.(Bellg, et al., 2004) We recommend that investigators describe fidelity strategies specific to two areas, provider training and treatment delivery, when reporting interventions. Common fidelity strategies for treatment delivery are fully described in several publications, but these generally include the use of standardized training for interventionists, manualized protocols, and methods to monitor intervention delivery (Radziewicz, et al., 2009; Resnick, et al., 2005; Robb, Burns, Docherty, \& Haase, In Review). Fidelity strategies for intervention delivery are used to: 1) control for interventionist differences, 2) reduce differences within treatment, 3 ) ensure adherence to 
treatment protocols, and 4) minimize contamination between study conditions. These strategies are important because they help ensure that study conditions were delivered as intended; the omission of strategies from intervention reporting impedes evaluation about how investigators addressed potential threats to intervention reliability and validity (Bellg, et al., 2004; Borrelli, et al., 2005).

Item 4F: Setting. Describe where the intervention was delivered; include location, privacy level, and ambient sound.

An apparent and defining quality of music-based interventions is the use of auditory stimuli to achieve desired changes in physiological and psychological function, yet few investigators report detailed information about the setting where music-based interventions are delivered (Robb \& Carpenter, 2009). Understanding the sound environment, or setting, where music-based interventions are introduced is very important when interpreting and/or comparing study outcomes. Factors related to setting that may influence outcomes include the location where the intervention was delivered (e.g., a patient's room vs. post-operative recovery room), as well as the privacy level and amount of ambient sound in that location. For example, high levels of environmental noise or diminished speech privacy can increase distress and impede communication (Christensen, 2007; Joseph, 2007; Thomas \& Martin, 2000). For example, differences in outcomes may occur with a music imagery intervention targeting pre-operative anxiety when it is delivered in a patient's private room, where there is little to no ambient sound, compared to the same intervention delivered in a pre-operative area with several beds separated only by curtains and moderate ambient sounds coming from televisions, family/staff conversations, and monitoring equipment. Disparate or even similar outcomes between these studies would raise interesting questions about the degree to which setting factors are relevant to study outcomes. Similarly, information about the setting would inform translation of the intervention to clinical practice.

Item 4G: Unit of Delivery. Specify whether interventions were delivered to individuals or groups of individuals, including the size of the group.

Interventions can be delivered to individuals or groups of people. Although a majority of intervention reports specify the unit of delivery, this information is not always explicitly stated and in some instances it has been overlooked.(Robb \& Carpenter, 2009) Reasons for reporting unit of delivery include the possibility that social and cost-related benefits may occur when interventions are delivered to groups of people (Boyle, McCartney, Forbes, \& O'Hare; Craigie, Nathan, Craigie, \& Nathan, 2009; de Groot, et al., 2007; Dickson, et al., 2009; Osborn, et al., 2006; Valentine, 2001). Group interventions may have added value in terms of peer support, motivation through group accountability, and social interactions that may have effects above and beyond the intervention itself. Group interventions may also be less costly to implement if they prove to be efficacious. In order to determine the relevance of these and other factors, it is important that authors specify whether interventions were delivered to individuals or groups of individuals. When groups are the unit of delivery, authors should specify the number of individuals participating in each group.

\section{CLOSING REMARKS}

Clear, detailed intervention descriptions within research publications are essential to improve replication and translation of music-based interventions to clinical practice. Although previous reporting guidelines provide investigators with checklists for behavioral trials, music interventions have additional components that need to be reported to enhance transparency. We have identified and described major components of music-based interventions that we believe should be included in research reports. The main purpose of these guidelines and the corresponding checklist is to increase transparency. We do not suggest a particular order in which information should be reported; rather, our emphasis is 
on the inclusion of information essential to understanding and interpreting study outcomes. Though we have attempted to be complete in the identification and description of musicbased intervention content, it is impossible to capture all of the essential content investigators should report - ultimately this responsibility lies with the investigator and should be guided by the study's theoretical framework.

\section{Acknowledgments}

This paper was supported by Award Number R01NR008583 from the National Institutes of Health-National Institute of Nursing Research (Robb, Burns); U10 CA098543 and U10 CA095861from the National Cancer Institute (Robb, Burns); and R01CA132927 from the National Cancer Institute (Carpenter, Burns). This paper was also supported by the first author's institutional CTSI career development award, PHS (NCCR) KL2RR025760-02. The content is solely the responsibility of the authors and does not necessarily represent the official views of the National Institute of Nursing Research, National Cancer Institute, or the National Institutes of Health.

\section{References}

Allen K, Blascovich J. Effects of music on cardiovascular reactivity among surgeons. Journal of the American Medical Association. 1994; 272(11):882-884. [erratum appears in JAMA 1994 Dec 14;272(22):1724]. [PubMed: 7811324]

Altman DG, Schulz KF, Moher D, Egger M, Davidoff F, Elbourne D, et al. The revised CONSORT statement for reporting randomized trials: explanation and elaboration. Annals of Internal Medicine. 2001; 134(8):663-694. [PubMed: 11304107]

Bellg AJ, Borrelli B, Resnick B, Hecht J, Minicucci DS, Ory M, et al. Enhancing treatment fidelity in health behavior change studies: best practices and recommendations from the NIH Behavior Change Consortium. Health Psychology. 2004; 23(5):443-451. [PubMed: 15367063]

Borrelli B, Sepinwall D, Ernst D, Bellg AJ, Czajkowski S, Breger R, et al. A new tool to assess treatment fidelity and evaluation of treatment fidelity across 10 years of health behavior research. Journal of Consulting and Clinical Psychology. 2005; 73(5):852-860. [PubMed: 16287385]

Boutron I, Moher D, Altman DG, Schulz KF, Ravaud P, Group C. Extending the CONSORT statement to randomized trials of nonpharmacologic treatment: explanation and elaboration. Annals of Internal Medicine. 2008a; 148(4):295-309. [PubMed: 18283207]

Boutron I, Moher D, Altman DG, Schulz KF, Ravaud P, Group C. Methods and processes of the CONSORT Group: example of an extension for trials assessing nonpharmacologic treatments. Annals of Internal Medicine. 2008b; 148(4):W60-66. [PubMed: 18283201]

Boyle J, McCartney E, Forbes J, O'Hare A. A randomised controlled trial and economic evaluation of direct versus indirect and individual versus group modes of speech and language therapy for children with primary language impairment. Health Technology Assessment. 11(25):iii-iv. [PubMed: 17610807]

Buffum MD, Sasso C, Sands LP, Lanier E, Yellen M, Hayes A, et al. A music intervention to reduce anxiety before vascular angiography procedures. Journal of Vascular Nursing. 2006; 24(3):68-73. [PubMed: 16952777]

Cassileth BR, Vickers AJ, Magill LA. Music therapy for mood disturbance during hospitalization for autologous stem cell transplantation: A randomized controlled trial. Cancer. 2003; 98(12):27232729. [PubMed: 14669295]

Cepeda MS, Carr DB, Lau J, Alvarez H. Music for pain relief. Cochrane Database Syst Rev. 2006; 2:CD004843. [PubMed: 16625614]

Chlan L, Tracy MF, Nelson B, Walker J. Feasibility of a music intervention protocol for patients receiving mechanical ventilatory support. Alternative Therapies in Health \& Medicine. 2001; 7(6): 80-83. [PubMed: 11712475]

Christensen M. Noise levels in a general intensive care unit: a descriptive study. Nursing in Critical Care. 2007; 12(4):188-197. [PubMed: 17883600]

Clark M, Isaacks-Downton G, Wells N, Redlin-Frazier S, Eck C, Hepworth JT, et al. Use of preferred music to reduce emotional distress and symptom activity during radiation therapy. Journal of Music Therapy. 2006; 43(3):247-265. [PubMed: 17037953] 
Craigie MA, Nathan P, Craigie MA, Nathan P. A nonrandomized effectiveness comparison of broadspectrum group CBT to individual CBT for depressed outpatients in a community mental health setting. Behavior Therapy. 2009; 40(3):302-314. [PubMed: 19647531]

Davidson KW, Goldstein M, Kaplan RM, Kaufmann PG, Knatterud GL, Orleans CT, et al. Evidencebased behavioral medicine: What is it and how do we achieve it? Annals of Behavioral Medicine. 2003; 26(3):161-171. [PubMed: 14644692]

de Groot J, Cobham V, Leong J, McDermott B, de Groot J, Cobham V, et al. Individual versus group family-focused cognitive-behaviour therapy for childhood anxiety: pilot randomized controlled trial. Australian \& New Zealand Journal of Psychiatry. 2007; 41(12):990-997. [PubMed: 17999271]

Des Jarlais DC, Lyles C, Crepaz N. Improving the reporting quality of nonrandomized evaluations of behavioral and public health interventions: the TREND statement. American Journal of Public Health. 2004; 94(3):361-366. [PubMed: 14998794]

Dewing J, Dewing J. Caring for people with dementia: Noise and light. Nursing Older People. 2009; 21(5):34-38. [PubMed: 19534171]

Dickson K, Marshall M, Boyle J, McCartney E, O’Hare A, Forbes J, et al. Cost analysis of direct versus indirect and individual versus group modes of manual-based speech-and-language therapy for primary school-age children with primary language impairment. International Journal of Language \& Communication Disorders. 2009; 44(3):369-381. [PubMed: 18821106]

Dijkers M, Kropp GC, Esper RM, Yavuzer G, Cullen N, Bakdalieh Y. Quality of intervention research reporting in medical rehabilitation journals. American Journal of Physical Medicine \& Rehabilitation. 2002; 81(1):21-33. [PubMed: 11807328]

Dzewaltowski DA, Estabrooks PA, Klesges LM, Bull S, Glasgow RE, Dzewaltowski DA, et al. Behavior change intervention research in community settings: How generalizable are the results? Health Promotion International. 2004; 19(2):235-245. [PubMed: 15128715]

Ebneshahidi A, Mohseni M, Ebneshahidi A, Mohseni M. The effect of patient-selected music on early postoperative pain, anxiety, and hemodynamic profile in cesarean section surgery. Journal of Alternative \& Complementary Medicine. 2008; 14(7):827-831.

Enderlin CA, Richards KC. Research testing of tailored interventions. Research Theory and Nursing Practice. 2006; 20(4):317-324.

Evans D. The effectiveness of music as an intervention for hospital patients: A systematic review. Jouranl of Advanced Nursing. 2002; 37(1):8-18.

Fowler-Kerry S, Lander JR. Management of injection pain in children. Pain. 1987; 30(2):169-175. [PubMed: 3670868]

Gagnier JJ, Boon H, Rochon P, Moher D, Barnes J, Bombardier C. Reporting randomized, controlled trials of herbal interventions: an elaborated CONSORT statement. Annals of Internal Medicine. 2006; 144(5):364-367. [PubMed: 16520478]

Graven SN. Sound and the developing infant in the NICU: conclusions and recommendations for care. Journal of Perinatology. 2000; 20(8 Pt 2):S88-93. [Review]. [PubMed: 11190706]

Hatem TP, Lira PI, Mattos SS. The therapeutic effects of music in children following cardiac surgery. Journal of Pediatrics. 2006; 82(3):186-192.

Hayes A, Buffum M, Lanier E, Rodahl E, Sasso C, Hayes A, et al. A music intervention to reduce anxiety prior to gastrointestinal procedures. Gastroenterology Nursing. 2003; 26(4):145-149. [PubMed: 12920428]

Hernandez-Ruiz E, Hernandez-Ruiz E. Effect of music therapy on the anxiety levels and sleep patterns of abused women in shelters. Journal of Music Therapy. 2005; 42(2):140-158. [PubMed: 15913391]

Huron D. The ramp archetype and the maintanence of passive auditory attention. Music Perception. 1992; 10(1):83-92.

Joseph, A.; Ulrich, R. Sound control for improved outcomes in healthcare settings. 2007. (Issue Paper \#4), 1-14. Retrieved from http://www.healthdesign.org/research/reports/sound.php

Kang JG, Lee JJ, Kim da M, Kim JA, Kim CS, Hahm TS, et al. Blocking noise but not music lowers bispectral index scores during sedation in noisy operating rooms. Journal of Clinical Anesthesia. 2008; 20(1):12-16. [PubMed: 18346603] 
Knight WE, Rickard DN. Relaxing music prevents stress-induced increases in subjective anxiety, systolic blood pressure, and heart rate in healthy males and females. Journal of Music Therapy. 2001; 38(4):254-272. [PubMed: 11796077]

Kreuter MW, Skinner CS. Tailoring: What's in a name? Health Education Research. 2000; 15(1):1-4. [PubMed: 10788196]

Labbe E, Schmidt N, Babin J, Pharr M, Labbe E, Schmidt N, et al. Coping with stress: The effectiveness of different types of music. Applied Psychophysiology \& Biofeedback. 2007; 32(3-4):163-168. [PubMed: 17965934]

Lechago SA, Carr JE, Lechago SA, Carr JE. Recommendations for reporting independent variables in outcome studies of early and intensive behavioral intervention for autism. Behavior Modification. 2008; 32(4):489-503. [PubMed: 18525063]

Lee D, Henderson A, Shum D, Lee D, Henderson A, Shum D. The effect of music on preprocedure anxiety in Hong Kong Chinese day patients. Journal of Clinical Nursing. 2004; 13(3):297-303. [PubMed: 15009332]

Marks D. Editorial: How should psychology interventions be reported? Journal of Health Psychology. 2009; 14:475-489. [PubMed: 19383648]

Marks D. Publication guidelines for intervention studies in the Journal of Health Psychology. Journal of Health Psychology. 2010; 15:5-7.

Mayo-Wilson E. Reporting implementation in randomized trials: proposed additions to the consolidated standards of reporting trials statement. American Journal of Public Health. 2007; 97(4):630-633. [PubMed: 17329641]

Megel ME, Houser CW, Gleaves LS. Children's responses to immunizations: Lullabies as a distraction. Issues in Comprehensive Pediatric Nursing. 1998; 21(3):129-145. [PubMed: 10531890]

Moher D, Jones A, Lepage L. Use of the CONSORT statement and quality of reports of randomized trials: A comparative before-and-after evaluation. Journal of the American Medical Association. 2001; 285(15):1992-1995. [PubMed: 11308436]

Moncher F, P FJ. Treatment fidelity in outcome studies. Clinical Psychology Review. 1991; 11:247266.

Noguchi LK. The effect of music versus nonmusic on behavioral signs of distress and self-report of pain in pediatric injection patients. Journal of Music Therapy. 2006; 43(1):16-38. [PubMed: 16671836]

Okamoto H, Stracke H, Stoll W, Pantev C, Okamoto H, Stracke H, et al. Listening to tailor-made notched music reduces tinnitus loudness and tinnitus-related auditory cortex activity. Proceedings of the National Academy of Sciences of the United States of America. 107(3):1207-1210. [PubMed: 20080545]

Osborn RL, Demoncada AC, Feuerstein M, Osborn RL, Demoncada AC, Feuerstein M. Psychosocial interventions for depression, anxiety, and quality of life in cancer survivors: meta-analyses. International Journal of Psychiatry in Medicine. 2006; 36(1):13-34. [PubMed: 16927576]

Pelletier CL. The effect of music on decreasing arousal due to stress: a meta-analysis. Journal of Music Therapy. 2004; 41(3):192-214. [PubMed: 15327345]

Perera R, Heneghan C, Yudkin P. Graphical method for depicting randomised trials of complex interventions. British Medical Journal. 2007; 334(7585):127-129. [PubMed: 17235093]

Philbin MK, Klaas P. Evaluating studies of the behavioral effects of sound on newborns. Journal of Perinatology. 2000; 20(8 Pt 2):S61-67. [PubMed: 11190703]

Pothoulaki M, MacDonald R, Flowers P. Methodological issues in music interventions in oncology settings: A systematic literature review. The Arts in Psychotherapy. 2006; 33:446-455.

Potteiger JA, Schroeder JM, Goff KL. Influence of music on ratings of perceived exertion during 20 minutes of moderate intensity exercise. Perceptual \& Motor Skills. 2000; 91(3 Pt 1):848-854. [PubMed: 11153860]

Radziewicz RM, Rose JH, Bowman KF, Berila RA, O’Toole EE, Given B. Establishing treatment fidelity in a coping and communication support telephone intervention for aging patients with advanced cancer and their family caregivers. Cancer Nursing. 2009; 32(3):193-202. [PubMed: 19295420] 
Ragneskog H, Gerdner LA, Josefsson K, Kihlgren M. Probable reasons for expressed agitation in persons with dementia. Clinical Nursing Research. 1998; 7(2):189-206. [PubMed: 9633339]

Resnick B, Inguito P, Orwig D, Yahiro JY, Hawkes W, Werner M, et al. Treatment fidelity in behavior change research: a case example. Nursing Research. 2005; 54(2):139-143. [PubMed: 15778656]

Robb SL, Burns DS, Docherty S, Haase JE. Ensuring treatment fidelity in a multi-site behavioral interventin study: Implementing NIH Behavior Change Consortium Recommendations in the SMART Trial. In Review.

Robb SL, Carpenter JS. A review of music-based intervention reporting in pediatrics. Journal of Health Psychology. 2009; 14(4):490-501. [PubMed: 19383649]

Robb SL, Nichols RJ, Rutan RL, Bishop BL, Parker JC. The effects of music-assisted relaxation on pre-operative anxiety. Journal of Music Therapy. 1995; 32:2-21.

Santacroce SJ, Maccarelli LM, Grey M. Intervention fidelity. Nurs Res. 2004; 53(1):63-66. [PubMed: 14726779]

Sarkamo T, Tervaniemi M, Laitinen S, Forsblom A, Soinila S, Mikkonen M, et al. Music listening enhances cognitive recovery and mood after middle cerebral artery stroke. Brain. 2008; 131(Pt 3): 866-876. [PubMed: 18287122]

Schulz KF, Altman DG, Moher D. CONSORT 2010 Statement: Updated Guidelines for Reporting Parallel Group Randomized Trials. Annals of Internal Medicine. 2010

Silverman MJ. The influence of music on the symptoms of psychosis: a meta-analysis. Journal of Music Therapy. 2003; 40(1):27-40. [PubMed: 17590966]

Simcock XC, Yoon RS, Chalmers P, Geller JA, Kiernan HA, Macaulay W, et al. Intraoperative music reduces perceived pain after total knee arthroplasty: a blinded, prospective, randomized, placebocontrolled clinical trial. The Journal of Knee Surgery. 2008; 21(4):275-278. [PubMed: 18979928]

Sloane PD, Mitchell CM, Weisman G, Zimmerman S, Foley KM, Lynn M, et al. The Therapeutic Environment Screening Survey for Nursing Homes (TESS-NH): An observational instrument for assessing the physical environment of institutional settings for persons with dementia. The Journals of Gerontology, Seriesl B: Psychological Sciences and Social Sciences. 2002; 57(2):S6978.

Smolen D, Topp R, Singer L, Smolen D, Topp R, Singer L. The effect of self-selected music during colonoscopy on anxiety, heart rate, and blood pressure. [Clinical Trial Randomized Controlled Trial]. Applied Nursing Research. 2002; 15(3):126-136. [PubMed: 12173164]

Standley, J. Music research in medical/dental treatment: An update of a prior meta-analysis. In: Smith, D., editor. Effectiveness of music therapy procedures: Documentation of research and clinical practice. 3. Silver Spring, MD: American Music Therapy Association; 2000. p. 1-64.

Thaut, M. Neuropsychological processes in music perception and their relevance in music therapy. In: Unkefer, R., editor. Music therapy in the treatment of adults with mental disorders: Theoretical bases and clinical interventions. St. Louis, MO: MMB Music, Inc; 2002. p. 2-32.

Thaut, M. Rhythm, music, and the brain. New York, NY: Routledge; 2005.

Thomas KA, Martin PA. NICU sound environment and the potential problems for caregivers. Journal of Perinatology. 2000; 20(8 Pt 2):S94-99. [PubMed: 11190707]

Valentine EEC. The effects of solo singing, group singing, and swimming on mood and physiological indices. British Journal of Medical Psychology. 2001; 74:115-120.

van der Geer ER, Vink AC, Schols JM, Slaets JP, Schols JMGA, Slaets JPJ. Music in the nursing home: Hitting the right note! The provision of music to dementia patients with verbal and vocal agitation in Dutch nursing homes. International Psychogeriatrics. 2009; 21(1):86-93. [PubMed: 18947457]

Wang SM, Kulkarni L, Dolev J, Kain ZN. Music and preoperative anxiety: A randomized, controlled study. Anesthesia \& Analgesia. 2002; 94(6):1489-1494. [PubMed: 12032013]

\section{Biographies}

Sheri L. Robb, PhD, MT-BC, is an Associate Research Scientist at the Indiana University School of Nursing. 
Debra S. Burns, PhD, MT-BC, is an Assistant Professor in the Department of Music and Arts Technology, School of Engineering and Technology, Indiana University-Purdue University Indianapolis.

Janet S. Carpenter, PhD, RN, FAAN, is a Professor in the Department of Adult Health, School of Nursing, Indiana University. 


\section{Table 1}

Consort and Trend Guidelines for Intervention Reporting

\begin{tabular}{|l|l|}
\hline CONSORT Item & $\begin{array}{l}\text { [Description of] The interventions for each group with sufficient details to allow replication, including how and when they } \\
\text { were actually administered. }\end{array}$ \\
\hline TREND Item & $\begin{array}{l}\text { Details of the interventions intended for each study condition and how and when they were actually administered, } \\
\text { specifically including: content, delivery method, unit of delivery, deliverer, setting, exposure quantity and duration, time } \\
\text { span, and activities to increase compliance or adherence. }\end{array}$ \\
\hline
\end{tabular}


Table 2

Checklist for Reporting Music-based Interventions

Music-based Intervention Reporting Criteria

A: Intervention Theory

Provide a rationale for the music selected; specify how qualities and delivery of the music are expected to impact targeted outcomes.

\section{B: Intervention Content}

Provide precise details of the music intervention and, when applicable, descriptions of procedures for tailoring interventions to individual participants.

\section{B.1: Person Selecting the Music}

Specify who selected the music: (1) pre-selected by investigator, (2) participant selected from limited set, (3) participant selected from own collection, or (4) tailored based on patient assessment.

\section{B.2: Music}

When using published music, provide reference for sheet music or sound recording.

When using improvised or original music, describe the music's overall structure (i.e., form, elements, instruments, etc).

\section{B.3. Music Delivery Method (Live or Recorded)}

When using live music, specify who delivered the music and the size of the performance group (e.g., interventionist only, interventionist and participant).

When using recorded music, specify placement of playback equipment and the use of headphones vs. speakers. Specify who determined/controlled volume (e.g., interventionist; participant. Specify decibel level of music delivered and/or use of volume controls to limit decibels.

\section{B.4: Intervention Materials}

Specify music and/or non-music materials.

\section{B.5: Intervention Strategies}

Describe music-based intervention strategies under investigation (examples: music listening, songwriting, improvisation, lyric analysis, rhythmic auditory stimulation, etc).

\section{C: Intervention Delivery Schedule}

Report number of sessions, session duration, and session frequency including practice sessions.

\section{D: Interventionist}

Specify interventionist qualifications and credentials.

Specify how many interventionists deliver study conditions.

\section{E: Treatment Fidelity}

Describe strategies used to ensure that treatment and/or control conditions were delivered as intended (e.g., interventionist training, manualized protocols, and intervention monitoring).

\section{F: Setting}

Describe where the intervention was delivered; include location, privacy level, and ambient sound.

\section{G: Unit of Delivery}

Specify whether interventions were delivered to individuals or groups of individuals, including the size of the group.

Note: This checklist may be reprinted and used without permission as a tool to help ensure transparent reporting of music-based interventions. Robb, S. L., Carpenter, J. S., Burns, D. S. (2010). Reporting guidelines for music-based interventions. Journal of Health Psychology, 16(2), 342-352 DOI: 10.1177/1359105310374781. 